\title{
Porcine Myeloid Antimicrobial Peptides: A Review of the Activity and Latest Advances
}

\author{
Shuaibing Shi ${ }^{1 \dagger}$, Tengfei Shen ${ }^{1 \dagger}$, Yongqing Liu ${ }^{1}$, Liangliang Chen ${ }^{1}$, Chen Wang ${ }^{1 *}$ and \\ Chengshui Liao ${ }^{1,2 *}$

\footnotetext{
${ }^{1}$ The Key Lab of Veterinary Biological Products, Henan University of Science and Technology, Luoyang, China, ${ }^{2}$ College of Animal Science and Technology/Luoyang Key Laboratory of Live Carrier Biomaterial and Animal Disease Prevention and Control, Henan University of Science and Technology, Luoyang, China
}

OPEN ACCESS

Edited by:

Chi-Chung Chou,

National Chung Hsing

University, Taiwan

Reviewed by:

Edwin Veldhuizen

Utrecht University, Netherlands

Charles Lee Bevins,

University of California, Davis,

United States

${ }^{*}$ Correspondence:

Chen Wang

wangchen2001@126.com

Chengshui Liao

liaochengshui33@163.com

tThese authors have contributed equally to this work

Specialty section: This article was submitted to Veterinary Pharmacology and

Toxicology

a section of the journa

Frontiers in Veterinary Science

Received: 04 February 2021

Accepted: 19 April 2021

Published: 14 May 2021

Citation:

Shi S, Shen T, Liu Y, Chen L, Wang C and Liao C (2021) Porcine Myeloid Antimicrobial Peptides: A Review of the Activity and Latest Advances.

Front. Vet. Sci. 8:664139.

doi: 10.3389/fvets.2021.664139
Traditional antibiotics have made great contributions to human health and animal husbandry since the discovery of penicillin in 1928, but bacterial resistance and drug residues are growing threats to global public health due to the long-term uncontrolled application of antibiotics. There is a critical need to develop new antimicrobial drugs to replace antibiotics. Antimicrobial peptides (AMPs) are distributed in all kingdoms of life, presenting activity against pathogens as well as anticancer, anti-inflammatory, and immunomodulatory activities; consequently, they have prospects as new potential alternatives to antibiotics. Porcine myeloid antimicrobial peptides (PMAPs), the porcine cathelicidin family of AMPs, have been reported in the literature in recent years. PMAPs have become an important research topic due to their strong antibacterial activity. This review focuses on the universal trends in the biochemical parameters, structural characteristics and biological activities of PMAPs.

Keywords: antimicrobial peptide, porcine, PMAPs, bacterial resistance, biological activity

\section{INTRODUCTION}

Since the discovery of antibiotics in the twentieth century, they have been widely used in human medicine and animal husbandry due to their antibacterial, antiviral, antitumour, and growth-promoting properties $(1,2)$. However, with the misuse of antibiotics, bacterial resistance, antibiotic pollution, and drug residues have become global public health problems (1-3). In the UK, approximately half of deaths due to Gram-negative bacterial infection are attributed to the development of bacterial resistance (4). If the problem of antimicrobial resistance is not addressed, some common bacterial infectious diseases can threaten people's lives again (5). A total of 23,000 people die each year from antimicrobial-resistant pathogens, and the number will increase to 10 million by $2050(6,7)$. Gross domestic product will decline by $\sim 2-3.5 \%$ per year $(8)$, and the economic loss caused by bacterial drug resistance may reach US $\$ 2.9$ trillion per year (9). Because the development of new antimicrobials has always been slower than the evolution of drug-resistant bacteria (4), we urgently need to research a new antimicrobial drug to replace antibiotics.

\section{ANTIMICROBIAL PEPTIDES}

Antimicrobial peptides (AMPs) are effector molecules of the innate immune defense system of animals and plants (10). AMPs have attracted extensive attention from scientists due to their low molecular weight (generally 30-60 amino acids), broad antimicrobial spectrum, and lack of 
induction of drug resistance (7). AMPs selectively kill bacteria, fungi, viruses, and even tumors (9), and they are a potential substitute for traditional antibiotics and are expected to be new antimicrobial drugs used in clinical applications (11). During the 1980s, the first cecropins were isolated by Hans Boman in the chrysalis of Hyalophora cecropia (12). AMPs have been found in vertebrates, invertebrates, amphibians, plants, marine organisms, fungi, bacteria, and other organisms $(13,14)$. AMPs are mainly divided into four categories according to the source as follows: animal-derived AMPs, plant-derived AMPs, microbial-derived AMPs, and synthetic AMPs $(15,16)$. Almost every class of AMPs comprises the cathelicidin family and the defensin family, which are the two largest groups of all AMPs (17). The cathelicidin family has strong antibacterial activities $(18,19)$, low haemolytic activity, and high cytotoxicity to drug-resistant strains $(20,21)$.

The cathelicidin family of porcine AMPs includes four categories as follows: PR-39, PG1-5 (protegrin), PF1-2 (prophenin), and porcine myeloid antimicrobial peptides (PMAPs) (22). PR-39 was initially isolated and purified from the porcine small intestine and has a type II poly-L-proline helix. PR39 is a cationic host defense peptide rich in proline and arginine with a molecular weight of $\sim 4,700 \mathrm{Da}$ (23-25). PGl-5 was originally isolated from porcine leukocytes and is rich in cysteine and arginine with a molecular weight of $\sim 2,000 \mathrm{Da}(26,27)$, and PGl-5 has $\beta$-hairpin structures. PF1-2 was initially isolated from porcine neutrophils and has an extended helical structure rich in proline and phenylalanine, with a molecular weight of $\sim 8,000 \mathrm{Da}(28,29)$. PMAPs originate from porcine bone marrow cells and are stored in peripheral polymorphonuclear neutrophil granules in the constitutive form of a prepeptide (22, 30-32). PMAPs exhibit high antimicrobial activity and have a stable $\alpha$-helix structure (33-38). Among natural AMPs, $\alpha$-helical structures are the most prevalent and are the same as those in the cathelicidin family AMPs. AMPs with $\alpha$-helical structures generally exhibit broad-spectrum antibacterial activity, including activity against several clinically antibiotic-resistant strains, such as methicillin-resistant Staphylococcus aureus, vancomycinresistant Enterococcus faecalis, and multiresistant Pseudomonas aeruginosa (39). However, AMPs that lack the $\alpha$-helical structures of the porcine cathelicidin family, such as PR-39, are bactericidal against several Gram-negative bacteria (Escherichia coli and Salmonella typhimurium) and Gram-positive bacteria (Bacillus megatherium and Bacillus subtilis) (40), but Proteus vulgaris, $P$. aeruginosa, and S. aureus are resistant to PR-39 (41). In addition, PMAPs have the same carpet-like antibacterial mechanism. PMAPs carry many positive charges and easily bind to the negatively charged surface of bacterial membranes, and their binding power to eukaryotic cell membranes is weak or nonexistence, resulting in a relatively low haemolytic effect (42). PMAPs have similar antibacterial activity with high-efficiency and broad-spectrum (22). Thus, PMAPs are of great importance in the development of stable, safe, and efficient AMPs of the porcine cathelicidin family.

In recent years, our group has performed research on the structural modification and antimicrobial activity of PMAPs. According to the structure and mechanism of PMAPs, many scholars have designed multiple novel modified peptides with increased stability and antimicrobial activity (43-50). This review mainly summarizes the research progress on the structural characteristics and biological activities of PMAPs in recent years.

\section{PORCINE MYELOID ANTIMICROBIAL PEPTIDES}

PMAPs (PMAP-23, PMAP-36, and PMAP-37), a class of cationic AMPs derived from porcine bone marrow cells, are obtained by first extracting RNA from porcine bone marrow cells and then by cDNA cloning, and they can also be obtained by solidphase synthesis (31). The three peptides are amphiphilic with $\mathrm{N}$-terminal hydrophilicity and C-terminal hydrophobicity, and their sequence similarity is much lower than that of other peptides in the porcine cathelicidin family. PR-39 has seven repeats of Xaa-Pro-Pro-Xaa, and PFs have several repeats of the Phe-Pro-Pro-Pro-Asn-Phe-Pro-Gly-Pro-Arg decamer. Only one to two amino acids differ in PGs, and the other amino acids are the same between them. In addition, the rank order of the peptides is similar (22). The biochemical parameters of PMAPs are shown in Table 1. The helical wheel diagrams of PMAPs are shown in Figures 1-3. The three peptides have an $\alpha$-helical structure with no toxic side effects to mammalian cells (51). In addition, the $\alpha$-helix structure, which exists in the secondary structure of various peptides, is an excellent amphiphilic structure with a positively charged hydrophilic side and a negatively charged hydrophobic symmetric side $(52,53)$.

PMAP-23 is an $\alpha$-helical antimicrobial peptide consisting of 23 amino acid residues with a molecular weight of 2962.63 Da, and the amino acid sequence of PMAP-23 is RIIDLLWRVRRPQKPKFVTVWVR (44, 54). PMAP23 contains seven highly positively charged amino acids (arginines and lysines) and 11 highly hydrophobic residues (55). PMAP-23 also contains two tryptophans with native fluorescence characteristics, and it can be used as a model to observe polypeptide-membrane interactions (56). The peptide possesses a unique helix-hinge-helix structure in the membrane environment, and the $\alpha$-helix structure is connected by a flexible hinge containing the PXXP motif $(35,57)$. PMAP-23 has two $\alpha$-helices in the $\mathrm{N}$-terminal region $(\operatorname{Arg} 1$ to $A r g 10)$ and the C-terminal region (Phe18 to Arg23) (55). The kink region consisting of two proline residues at positions 12 and 15 increases the amphiphilicity of the charged residues (33). PMAP-23 easily binds to bacterial membranes containing many negatively charged lipids by electrostatic adsorption. Therefore, PMAP-23 has a certain selectivity for the target cell membrane. The bactericidal mechanism of PMAP-23 is a carpet-like mechanism (58). Natural and synthetic PMAP-23 show antibacterial activity against Gram-positive bacteria, Gram-negative bacteria, and fungi $(35,55)$.

PMAP-36 is a highly cationic amphiphilic $\alpha$-helical antimicrobial peptide consisting of 36 amino acid residues with a molecular weight of $4157.22 \mathrm{Da}(47,59)$, and the complete amino acid sequence of PMAP-36 is GRFRRLRKKTRKRLKK IGKVLKWIPPIVGSIPLGCG (59). PMAP-36 has the highest net charge with 13 positively charged amino acids (seven lysines and 
TABLE 1 | Biochemical parameters and structures of PMAPs.

\begin{tabular}{|c|c|c|c|c|c|c|c|}
\hline Peptide & Sequence & Formula & $\mathbf{M W}^{\mathbf{a}}$ & Charge & $\mathbf{P I}^{\mathrm{b}}$ & GRAVYc & Structure \\
\hline PMAP-23 & RIIDLLWRVRRPQKPKFVTVWVR & $\mathrm{C}_{140} \mathrm{H}_{229} \mathrm{~N}_{43} \mathrm{O}_{28}$ & 2962.63 & +7 & 12.18 & -0.296 & $\alpha$-helical \\
\hline PMAP-36 & GRFRRLRKKTRKRLKKIGKVLKWIPPIVGSIPLGCG & $\mathrm{C}_{191} \mathrm{H}_{336} \mathrm{~N}_{62} \mathrm{O}_{39} \mathrm{~S}_{1}$ & 4157.22 & +13 & 12.31 & -0.461 & $\alpha$-helical \\
\hline PMAP-37 & GLLSRLRDFLSDRGRRLGEKIERIGQKIKDLSEFFQS & $\mathrm{C}_{192} \mathrm{H}_{320} \mathrm{~N}_{60} \mathrm{O}_{56}$ & 4365.02 & +9 & 10.24 & -0.724 & $\alpha$-helical \\
\hline
\end{tabular}

${ }^{a}$ Molecular weight; ${ }^{b}$ Theoretical isoelectric point; ${ }^{c}$ Grand average of hydropathicity.

a, b, c, were calculated by the antimicrobial peptide analysis tool. (https://web.expasy.org/protparam/).

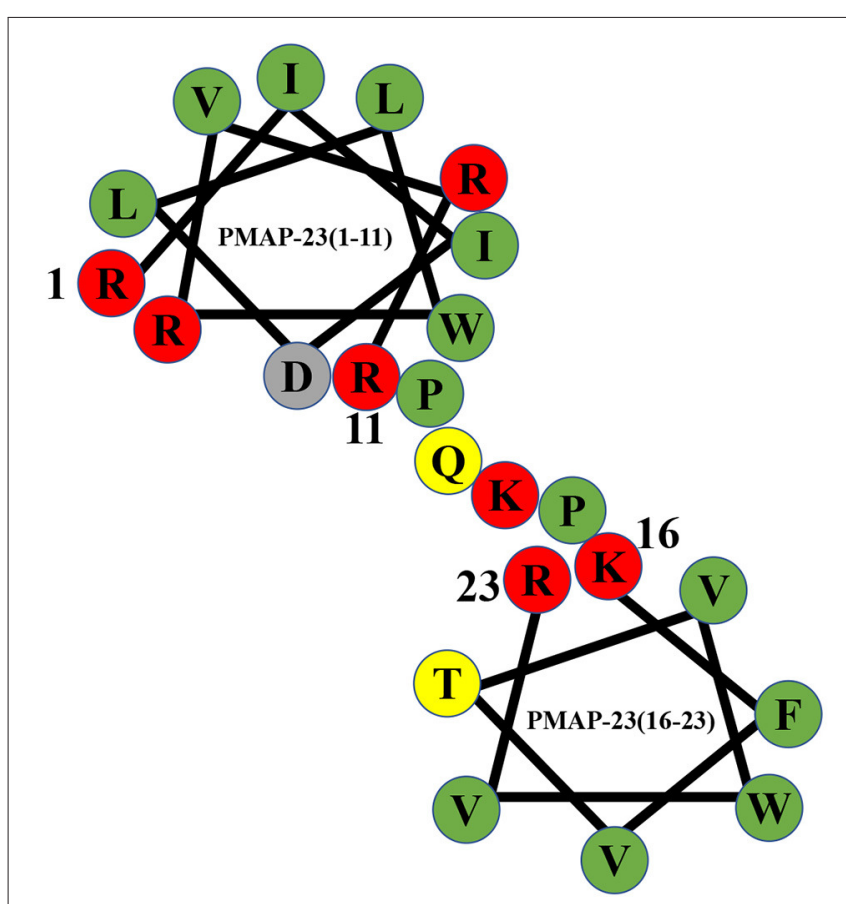

FIGURE 1 | Helical wheel diagram of PMAP-23. Hydrophobic residues are indicated by green circles, and positively charged residues are indicated by red circles. Negatively charged residues are indicated by gray circles, and neutral uncharged residues are indicated by yellow circles. The diagram clearly shows that PMAP-23 has a helix-hinge-helix structure.

six arginines). Cationic amino acids are mostly in the $\mathrm{N}$-terminus and form an $\alpha$-helical structure at the $\mathrm{N}$-terminus. The high net charge contributes to the electrostatic interaction between PMAP-36 and negatively charged molecules on the surface of bacterial cell membranes $(60,61)$. The peptide also contains 21 hydrophobic amino acids in the C-terminus (34). The highly hydrophobic tail plays an important role in broad-spectrum antimicrobial activity $(61,62)$. The C-terminal 35 th amino acid of PMAP-36 is a cysteine, which can dimerize two PMAP-36 peptides through intermolecular disulphide bonds, and these peptides are stored in leukocytes as dimers (62). Although monomers and dimers show rapid and effective bactericidal activity against most microorganisms, dimeric peptides have a more stable conformation, longer bacteriostatic time, and lower medium sensitivity $(34,62)$.

PMAP-37 is an amphiphilic antimicrobial peptide that contains 37 amino acid residues with a molecular weight of
4365.02 Da $(38,50)$, and the amino acid sequence of PMAP37 is GLLSRLRDFLSDRGRRLGEKIERIGQKIKDLSEFFQS (48, 49). PMAP-37 has the highest hydrophobicity and the lowest net charge (22). In trifluoroethanol, PMAP-37 can form an $\alpha$-helixdominated structure from an irregular coiled structure, and it is a typical amphiphilic $\alpha$-helix cationic antimicrobial peptide (38). Moreover, PMAP-37 is the strongest membrane-active peptide, which can permeate the intracellular membrane of bacteria at $0.2-1 \mu \mathrm{M}(37,63)$. The $15-32$ stretch of PMAP-37 is significantly similar to the N-terminal extension of cecropin B and A in Drosophila melanogaster and Cecropia hyalophora, respectively (38). Therefore, PMAP-37 provides a unique example of a sequence convergence structure for the scientific community.

\section{BIOLOGICAL ACTIVITY OF PMAPS}

\section{Antibacterial Activity Antibacterial Mechanism of PMAPs}

AMPs can interact with the cell membrane of bacteria and then destroy the integrity of the membrane, ultimately leading to leakage of the cell contents and killing the cells $(13,53)$. Various models for the interaction of AMPs with cell membranes have been proposed, such as barrel-stave, carpet-like, and toroidalpore models $(13,35,64)$, and the methods of damaging the membrane are different in each model. For example, in the barrel-stave model, AMPs insert into the cell membrane phospholipid bilayer from a direction perpendicular to the cell membrane as multimers by polymerizing with the phospholipid bilayer, thereby forming ion channels across the cell membrane that rupture the membrane. In the carpet-like model, AMPs saturate the membrane and then disrupt the cell membrane (64). Here, we mainly introduce the mechanism of the carpet-like model of PMAPs $(38,58,61,65)$ (Figure 4). AMP molecules aggregate on the surface of the membrane in large quantities and remain in equilibrium with the membrane through electrostatic interactions, forming a carpet-like morphology (66). The peptide then begins to reorient. The hydrophobic region of the peptide is oriented preferentially toward and bound to the phospholipid head groups of the membrane, and its hydrophilic region is oriented toward the solvent. Because the peptide consistently makes contacts with the phospholipid head during the action, there is no need to insert into the hydrophobic core of the membrane. When the peptide reaches a threshold concentration, the membrane fluidity changes until the membrane structure is unstable. The peptide penetrates the membrane, or the membrane disintegrates into micelles, allowing the cellular contents to extravasate and ultimately leading to bacterial death 

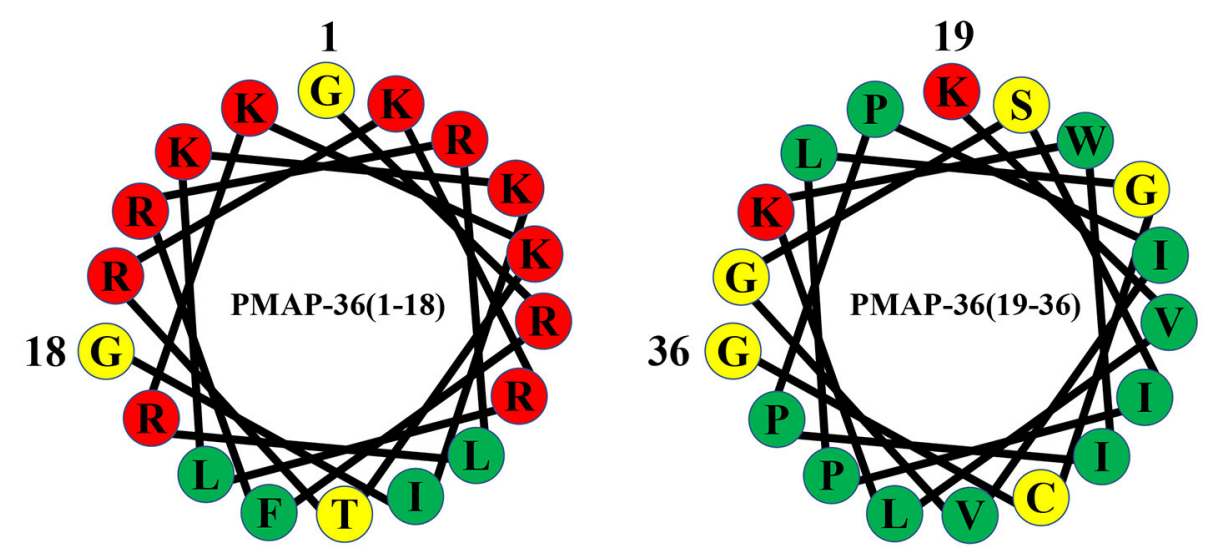

FIGURE 2 | Helical wheel diagram of PMAP-36. Hydrophobic residues are indicated by green circles, and positively charged residues are indicated by red circles. Negatively charged residues are indicated by gray circles, and neutral uncharged residues are indicated by yellow circles.
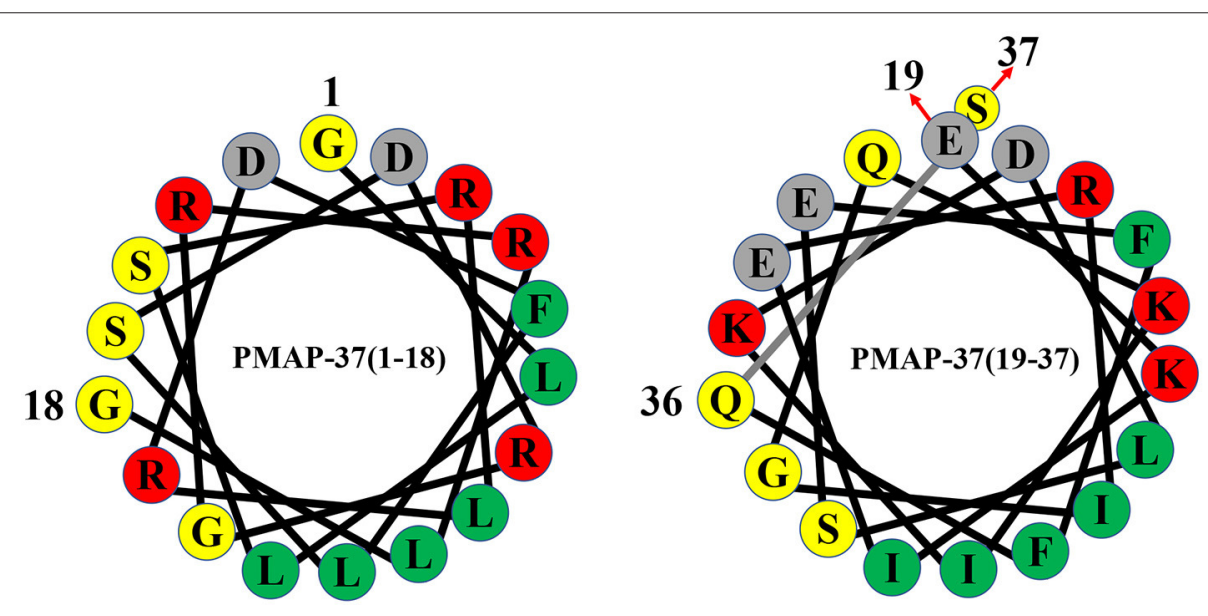

FIGURE 3 | Helical wheel diagram of PMAP-37. Hydrophobic residues are indicated by green circles, and positively charged residues are indicated by red circles. Negatively charged residues are indicated by gray circles, and neutral uncharged residues are indicated by yellow circles.

(67-69). Because transmembrane pores may also form when the concentration of some peptides is below a threshold, the mechanism by which AMPs disrupt the membrane has an important relationship with the concentration $(13,35,64)$. PMAPs increase the contents of $\mathrm{NO}$ and reactive oxygen species (ROS) in E. coli cells, causing DNA strand oxidative breaks and cell death. In addition, DNA damage is also part of the bacterial death mechanism (70).

\section{Antibacterial Activity of PMAP-23}

PMAP-23 has high antimicrobial activity against Gramnegative and Gram-positive bacteria (71). Compared to the antimicrobial activity of the conventional ampicillin antibiotic, the antimicrobial activities of PMAP-23 and PMAP-23N (Cterminal amidated) against Gram-negative bacteria are 10- to 80 -fold higher (72). However, the MIC of PMAP-23 against $S$. aureus is slightly higher than that against other bacteria (56). The N-terminus of PMAP-23 is significantly associated with its antimicrobial activity, but salt tolerance is dependent on the C-terminal amino acids (33). $\mathrm{NaCl}$ has an inhibitory effect on the activity of the truncated peptide of PMAP-23 (1-17, 73), which may be due to the reduction in positively charged amino acids and the consequent decrease in electrostatic interactions between the polypeptide and the bacterial membrane (74), and the stability of the $\alpha$-helix structure is also essential for antibacterial activity (75). The reduced activity of truncated peptides may be related to the disruption of the $\alpha$-helix structure. In the C-terminal truncated peptide of PMAP $(1-13,73)$, the antimicrobial activity is enhanced after replacing the negatively charged aspartic acid with a positively charged lysine, which may be explained by the fact that increasing the net charge of the polypeptide enhances the antibacterial activity (76). Indeed, the principle applies equally to helical AMPs of other cathelicidin families. For example, cathelicidin-BF (Cath-BF) is a helical AMP extracted from Bungarus fasciatus and contains 34 amino acid residues. Cath- $\mathrm{A}$ and Cath-B, two analogs 


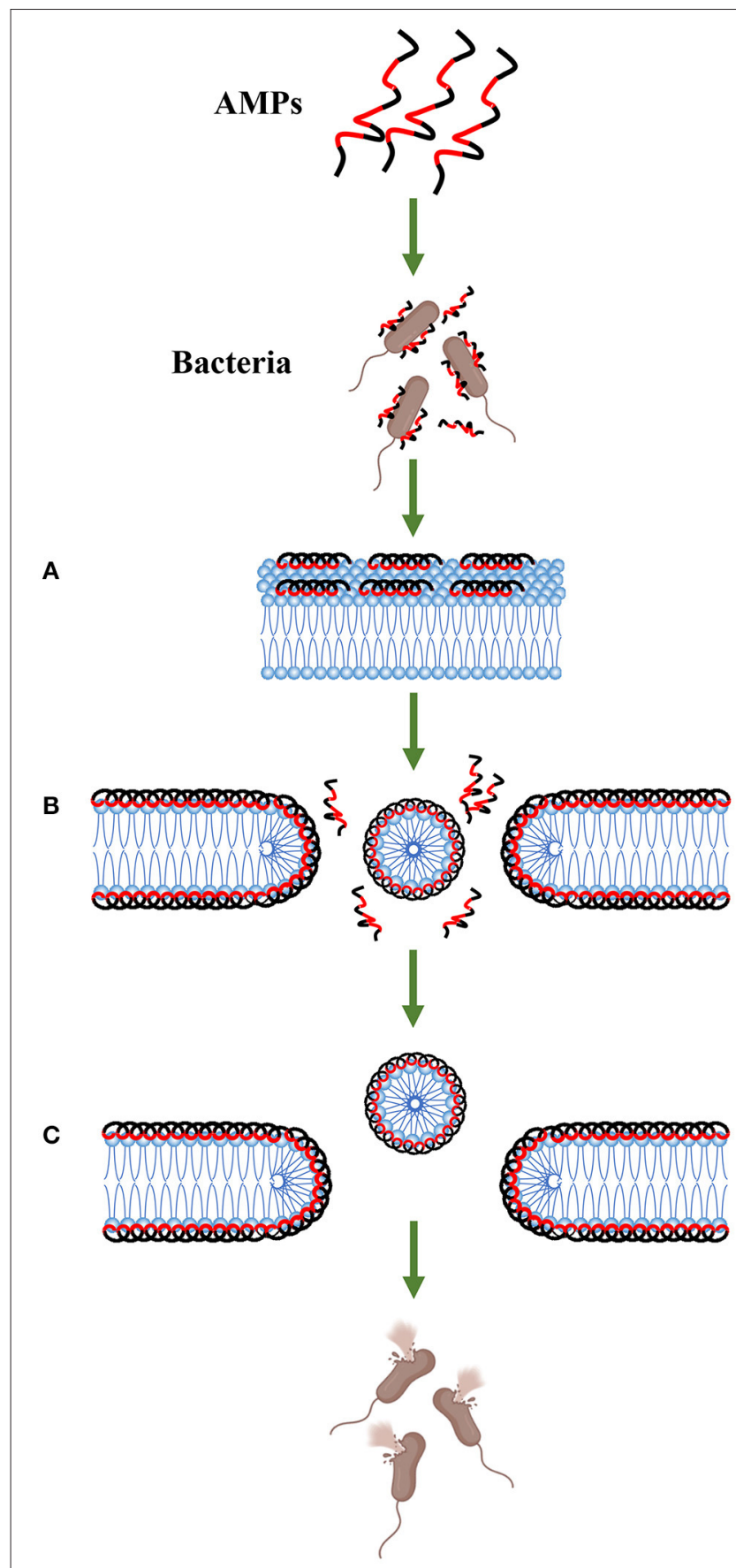

FIGURE 4 | Carpet-like mechanism. Antimicrobial peptides aggregate on the surface of the bacteria and remain in equilibrium with the membrane through electrostatic interactions. The hydrophobic surface (red area) faces the membrane, and the hydrophilic surface (black area) faces the solvent (step A). When the peptide monomer reaches a threshold concentration, the membrane is permeated, and a temporary pore is formed (step B). This process may also cause the membrane to disintegrate into micelles (step C).

of Cath-BF, have positively charged lysine substitutions that increase the net charge by three and two, respectively. Among them, Cath-A exhibits 2-fold greater antibacterial activity against methicillin-resistant $S$. aureus than the parent Cath-BF peptide. For E. coli ATCC 25922, although the antibacterial activity of Cath-A is similar to that of Cath-BF, the activity of Cath-B is enhanced by 4 -fold (77). CRAMP-18 is an AMP derived from the murine cathelicidin family, and the $\mathrm{K}^{2}$-CRAMP-18 derivative peptide has an increased net charge. The antimicrobial activity of $\mathrm{K}^{2}$-CRAMP-18 against Gram-negative bacteria (such as E. coli and S. Typhimurium), Gram-positive bacteria (such as $S$. pyogenes and S. aureus), and fungi (such as Albicans) is increased by 8 - to 16 -fold with almost no haemolytic activity. Therefore, the potential antibacterial activity of $\mathrm{K}^{2}$-CRAMP-18 is enhanced by increasing the positive net charge of AMPs (78).

The fifth amino acid (leucine) of the PMAP-23-derived peptides, PMAP-23R (Leu5-Arg), PMAP-23I (Thr19-Ile), and PMAP-23RI (Leu5-Arg and Thr19-Ile), is substituted with an arginine to increase the net charge, and the nineteenth amino acid (threonine) of PMAP-23 is substituted with an isoleucine to increase the hydrophobicity (43). Both the antimicrobial spectrum and activity of PMAP-23 are significantly increased, especially against Gram-negative bacteria. PMAP-23R has a higher antimicrobial activity than PMAP-23, which may be due to the increase in positive charge, allowing PMAP-23R to more easily bind to negatively charged bacterial membranes (79). The increased antimicrobial activity of PMAP-23I is perhaps due to the increased hydrophobicity, which in turn also increases the affinity of PMAP-23I for membranes, allowing it to be more easily entrapped in the phospholipid layer of bacteria (80, 81). PMAP-23RI has an increased net charge and hydrophobicity, resulting in the best antibacterial effect. Similar to PMAP-23, human LL-37 is an $\alpha$-helical AMP from the cathelicidin family. Nagaoka utilized the amphipathic 18mer peptide $\left(\mathrm{K}^{15}-\mathrm{V}^{32}\right)$ of $\mathrm{LL}-37$ as a template and designed a new peptide, 18-mer LL, by enhancing its hydrophobicity using two $\mathrm{L}$ residues to replace $\mathrm{E}^{16}$ and $\mathrm{K}^{25}$ followed by the replacement of $\mathrm{Q}^{22}, \mathrm{D}^{26}$, and $\mathrm{N}^{30}$ of the 18 -mer $\mathrm{LL}$ with three $\mathrm{K}$ residues to enhance its positive charge to create the derivative peptide, 18-mer LLKKK. Compared with the parental 18-mer peptide, 18-mer LL has enhanced hydrophobicity and better antibacterial efficacy against both Gram-positive (such as MRSA and S. pyogenes) and Gram-negative bacteria (such as E. coli and $P$. aeruginosa); however, the 18-mer LLKKK peptide, which has both increased hydrophobicity and positive charge, exhibits the best antibacterial efficacy (82). Therefore, the antimicrobial activity of AMPs is enhanced by increasing their hydrophobicity and net charge. Similarly, fatty acid modification with hydrophobicity can also serve as a strategy to improve antibacterial activity. Fatty acid modification of a certain chain length (carbon number 4-14) enhances the hydrophobicity of the polypeptide, which enhances the antimicrobial activity (83). PMAP-23RI-Dec was designed by modifying the C-terminus of PMAP-23RI with decanoic acid. The antibacterial activities of PMAP-23RI-Dec are 4-fold higher than those of PMAP23RI (except against $P$. aeruginosa). In addition, mouse models of wound infection or an abscess caused by $S$. aureus and $P$. aeruginosa bacteria have been treated by subcutaneous injection of AMPs or antibiotics into mice, respectively, demonstrated that PMAP-23RI-Dec is more effective in treating infected mice (44). 


\section{Antibacterial Activity of PMAP-36}

PMAP-36 is an effective antimicrobial peptide against both Gram-positive and Gram-negative bacteria (34). In general, S. typhimurium is susceptible to most AMPs, but PMAP-36 has relatively low activity. However, PMAP-36 has high antimicrobial activity against $P$. aeruginosa, Bacillus megaterium, and $S$. aureus (84). In addition, PMAP-36 activity has been compared to the antibacterial activity of two widely studied polypeptides, human LL-37 and chicken Cath-2. PMAP-36 shows the strongest antimicrobial activity against $E$. coli $\mathrm{O} 78$ and has a significant inhibitory effect on E. coli $\mathrm{O} 78$ at $2.5 \mu \mathrm{M}(60)$.

The 24 residue truncated peptide, GI24, which contains all cationic amino acids of PMAP-36, has antibacterial activity consistent with that of PMAP-36 (61), demonstrated that the antibacterial activity of PMAP-36 is closely related to the positively charged N-terminal amino acids. The RI21 and RI18 truncated peptides of PMAP-36 also show good antibacterial activity against bacteria with higher antibacterial activity than melittin and significantly lower haemolytic activity than PMAP36 and melittin. In addition, both PMAP-36 and its derived peptides have potent antimicrobial activity against $B$. subtilis (36). Therefore, reducing the chain length of PMAP-36 within a certain range not only maintains antimicrobial activity but also reduces haemolysis.

PMAP-36 shows antimicrobial activity against both Gramnegative and Gram-positive bacteria, and it is 4- to 128 -fold more effective than gentamicin and streptomycin. In addition, antibiotic-peptide combinations have good antimicrobial activity against E. coli ATCC 25922 and S. aureus ATCC 29213. When PMAP-36 is combined with gentamicin, it has a synergistic effect on E. coli and a partial synergistic effect on S. aureus (59). Therefore, the synergistic antimicrobial activity of the peptide and antibiotics is a good prospective application.

PMAP-36PW and PMAP-36PK are two new peptides designed by replacing the prolines at positions 25 and 26 of PMAP-36 with tryptophan and lysine, which increases the hydrophobicity and positive charge as well as extends the $\alpha$ helical structure of PMAP-36. PMAP-36 has no bacteriostatic effect on E. coli K88, while PMAP-36PW and PMAP-36PK have an antibacterial effect on E. coli K88. Compared to that of PMAP-36, the antibacterial activities of PMAP-36PW and PMAP-36PK against Gram-positive bacteria and Gram-negative bacteria are also significantly increased, especially against Listeria monocytogenes, with increases in antibacterial activity of 4- to 8fold. In addition, in murine animal model experiments of acute infection with Salmonella choleraesuis and L. monocytogenes, the modified peptide shows therapeutic efficacy comparable to that of the ceftiofur sodium antibiotic (45). Myristic acid has been used to modify the N-terminus of PMAP-36PW to improve hydrophobicity, resulting in a novel modified peptide, Myr-36PW. Compared with PMAP-36PW, Myr-36PW has a significantly lower MIC against Gram-negative bacteria and Gram-positive bacteria, especially L. monocytogenes CICC 21634. The MIC of Myr-36PW is reduced by 4 -fold, and it has effective antibiofilm activity. In addition, a previous study investigating the effects of Myr-36PW in mice infected with $S$. aureus ATCC 25923 and P. aeruginosa GIM1.551, Myr-36PW showed a prominent therapeutic effect on mouse pneumonia and peritonitis experiments compared with those of PMAP$36 \mathrm{PW}$, and it promoted abscess reduction and wound healing in infected mice. Compared to the penicillin antibiotic, there was no significant difference in the therapeutic effect (47). This study provides an important reference value for the development of novel antimicrobial drugs.

\section{Antibacterial Activity of PMAP-37}

PMAP-37 has potent antimicrobial activity against both Grampositive (such as $B$. megaterium and $S$. aureus) and especially Gram-negative bacteria (such as E. coli, S. typhimurium, and $P$. aeruginosa) (38). Generally, the antimicrobial activity of AMPs is enhanced by modifying the primary and secondary structures of AMPs in a certain range to increase the net charge of AMPs, prolong their $\alpha$-helix, and increase their hydrophobicity (8588). PMAP-37 (R13-I) and PMAP-37 (K20/27-I) are two analogs with substitutions of isoleucine for arginine at position 13 and isoleucine for lysine at positions 20 and 27, resulting in increased hydrophobicity of PMAP-37. The sensitivity of Gram-negative bacteria to PMAP-37 and its analogs is higher than that of Grampositive bacteria, and the MIC of PMAP-37 (R13-I) for Gramnegative bacteria is lower than that of PMAP-37. Moreover, PMAP-37 (K20/27-I) has antimicrobial activity against Shigella flexneri CICC 21534, especially S. typhimurium SL1344, with $\sim 4$-fold increases in antimicrobial activity, and it has obvious therapeutic effects on mice infected with S. aureus ATCC 25923 and S. typhimurium SL1344 (48). The antimicrobial activity can be enhanced by increasing the net charge of the polypeptide to a certain range (89). Three PMAP-37 analogs, PMAP-37 (F9-R), PMAP-37 (F34-R), and PMAP-37 (F9/34-R), were designed by replacing the phenylalanine at positions 9 and 34 of PMAP-37 with arginine. In addition to the bacteriostatic activity against S. flexneri CICC 21534, PMAP-37 has higher bacteriostatic activity against other Gram-negative bacteria tested. Compared to PMAP-37, PMAP-37 (F9-R) and PMAP-37 (F9/34-R) have stronger antimicrobial activity against S. flexneri CICC 21534, and PMAP-37 (F34-R) has the highest antimicrobial activity (49).

A novel AMP, Chol-37 (F34-R), was designed by modifying the N-terminus of PMAP-37 (F34-R) with cholesterol, which increases the hydrophobicity of the peptide. The MICs of the modified Chol-37 (F34-R) peptide against S. aureus ATCC 25923 and S. typhimurium SL1344 are 2-fold lower than those against PMAP-37 (F34-R) and 4-fold lower than those of P. aeruginosa GIM1.551 and L. monocytogenes CICC 21634, and Chol-37 (F34-R) exhibits effective antibiofilm activity, low toxicity, and no haemolytic activity. In addition, in the model of knife injury and abscess, Chol-37 (F34-R) shows better wound healing and abscess reduction as well as a stronger ability to remove bacteria. In the peritonitis model infected with $S$. aureus ATCC 25923, the in vivo treatment and pathological injury recovery of Chol-37 (F34-R) are similar to those of benzylpenicillin potassium, surpassing ampicillin sodium; Chol-37 (F34-R) also shows strong antimicrobial activity, suggesting good application prospects (50). 
Similar to PMAP-23 and PMAP-36, PMAP-37 exhibits a broad antimicrobial spectrum with MICs of $\sim 1-4 \mu \mathrm{M}$. In addition, PMAP-37 also has haemolytic activity, and it has a haemolytic effect on human red blood cells at 10-15 $\mu \mathrm{M}$ (38). However, PMAP-23 and PMAP-36 do not lyse erythrocytes at concentrations higher than $100 \mu \mathrm{M}(31,38)$. At present, there are relatively few studies on PMAP-37.

\section{Antifungal Activity}

\section{Antifungal Activity of PMAP-23}

The antifungal mechanism of AMPs results in the loss of fungal cell activity mainly caused by the formation of transmembrane channels, which increases membrane permeability, leading to the destruction of microbial cell structure (90, 91). Kim studied the antifungal mechanism of PMAP-23 against Candida albicans at low concentrations, demonstrating that the concentration of PMAP-23 that causes bacterial membrane depolarization and potassium ion efflux $(2.5 \mu \mathrm{M})$ does not disrupt the cell membrane. Lower concentrations of PMAP-23 result in higher levels of calcium ions in the cytoplasm and mitochondria, which causes C. albicans apoptosis. Mitochondrial calcium-induced ROS are the main factors triggering apoptosis. Therefore, the antifungal mechanism of PMAP-23 not only destroys biofilms but also induces apoptosis (35).

PMAP-23 has significant antifungal activity against Saccharomyces cerevisiae KCTC 7296, Trichosporon beigelii KCTC 7707, and C. albicans TIMM 1768. In particular, PMAP23 has the highest antifungal activity against T. beigelii KCTC 7707, higher antifungal activity against C. albicans TIMM 1768, and no haemolytic activity. In addition, PMAP-23 inhibits the apical growth of Trichophyton rubrum, which has an obvious antigrowth effect (92).

Based on substitution of tryptophan for amino acids 10, 13, or 14 of PMAP-23, several PMAP-23 analogs have been designed, resulting in enhanced hydrophobicity of the parent peptide. Among these, the analogous P6 peptide has tryptophan at positions 10, 13, and 14 of PMAP-23 and has strong fungicidal activity. Compared to PMAP-23 and other analogs, P6 has the strongest antifungal effect on C. albicans, and the MIC of P6 is decreased by 4 - to 8 -fold compared to that of PMAP-23. P6 destroys the hyphal morphology of $C$. albicans by destroying the cell membrane of fungi, thereby resulting in a fungicidal effect on spores and changing the morphology of C. albicans. Therefore, fungicidal activity also significantly increases by enhancing the hydrophobicity of PMAP-23 (93).

\section{Antifungal Activity of PMAP-36}

Both monomers and dimers of PMAP-36 have antifungal activity against C. albicans, while PMAP-36 (1-18, 73), a C-terminal truncated peptide of PMAP-36, has no antifungal activity against C. albicans. Thus, the C-terminus is important for PMAP-36 to exert antifungal activity (34). A series of truncated peptides of PMAP-36 have been designed by amino acid truncation, and the antifungal activity and cell selectivity of these peptides against Candida have been investigated and compared with those of melittin (36). RI18 has high antifungal activity and the highest selectivity index for fungi with a value 108-fold higher than that of PMAP-36. RI21 and RI18 have high antifungal activity against $C$. albicans with MICs $\sim 16-21$-fold higher than that of PMAP-36, and the haemolytic activities are lower than those of PMAP-36 and melittin. Of these, RI21 has the lowest MIC against Candida with a value $\sim 6$-fold lower than that of PMAP36. In addition, all the peptides showed strong antifungal activity against Candida tropicalis 2.1975 comparable to the antifungal activity of melittin (36), indicating that reducing the chain length of PMAP-36 not only maintains high antimicrobial activity but also reduces haemolysis.

\section{Antiparasitic Activity}

PMAP-23 not only has an inhibitory effect on bacteria and fungi but also has an inhibitory effect on the eggs and worms of Caenorhabditis elegans (94). C. elegans cells treated with PMAP23 and stained with propidium iodide have significantly higher fluorescence intensity than normal cells. Confocal microscopy has also confirmed that PMAP-23 penetrates the cell membrane and is present in the eggshell and plasma membrane of C. elegans. After $20 \mathrm{~h}$ of incubation, PMAP-23 is inactive on the eggs but active on the worms. When incubated for $60 \mathrm{~h}$, PMAP-23 shows some activity on the eggs. This result may be due to the presence of the shell layer in the eggs, which affects the hatching rate. When the concentration of PMAP-23 is $200 \mu \mathrm{M}$, the hatchability of worm eggs does not decrease, but when the concentration is $25 \mu \mathrm{M}$, the mobility of worms becomes highly sensitive $(94,95)$. Therefore, PMAP-23 may exert its anti-worm nodule role by destroying the cell membrane structure of C. elegans.

\section{Antitumour Activity}

PMAP-23 shows some antitumour activity against both Jurkat and SNU 601 tumor cells, whereas P6 shows the highest antitumour activity with an activity $\sim 4$-fold that of PMAP23, confirming that increased hydrophobicity of PMAP-23 contributes to the antitumour activity (93). Shin SY designed two modified peptides, PMAP1 and PMAP2, by replacing proline at positions 12 and 15 of PMAP-23 with alanine, respectively, and investigated the antitumour activity of PMAP-23 and its engineered peptides in tumor cells (human chronic myeloid leukemia K-562 cells, human acute T-cell leukemia Jurkat cells, human lung cancer A-549 cells, and human breast cancer MDAMB-361 cells). The antitumour activity of PMAP-23 is 2-fold higher than that of PMAP1, while PMAP2 has no inhibitory effect on all tumor cells, indicating that the two prolines in PMAP23 play an important role in inhibiting tumor cells. In addition, the 15th proline in PMAP-23 is more important than the 12th proline in the antitumour effect, and PMAP-23 does not lyse human erythrocytes even at $100 \mu \mathrm{M}$ (96). The biological activities of PMAPs are summarized in Table 2.

\section{CONCLUSION AND FUTURE DIRECTION}

In the past 80 years, $\sim 5,000$ AMPs have been discovered or synthesized, and some of these are in preclinical and clinical development. Compared with antibiotics, AMPs have many advantages, such as broad antimicrobial spectrum, efficacy, selectivity, high potency, low toxicity, low accumulation in 
TABLE 2 | Biological activity of PMAPs.

\begin{tabular}{|c|c|c|c|}
\hline Peptide & Biological activity & Strains/parasites/tumor cells & References \\
\hline \multirow[t]{4}{*}{ PMAP-23 } & Antibacterial & $\begin{array}{l}\text { E. coli, S. typhimurium, P. aeruginosa, B. megaterium, } \\
\text { S. aureus, B. subtilis, Streptococcus pyogenes, } \\
\text { Bacillus globigii, P. vulgaris, Staphylococcus } \\
\text { epidermidis, L. monocytogenes, S. choleraesuis, } \\
\text { Pasteurella multocida, S. flexneri, Enterococcus } \\
\text { faecalis }\end{array}$ & $\begin{array}{l}(33,43,44,56,71,72 \\
76,79)\end{array}$ \\
\hline & Antifungal & C. albicans, T. beigelii, S. cerevisiae & $(35,92,93)$ \\
\hline & Antiparasitic & C. elegans & $(94,95)$ \\
\hline & Antitumour & SNU601, MDA-MB-361, Jurkat, A-549, K-562 & $(93,96)$ \\
\hline \multirow[t]{2}{*}{ PMAP-36 } & Antibacterial & $\begin{array}{l}\text { E. coli, S. typhimurium, P. aeruginosa, B. megaterium, } \\
\text { S. aureus, B. subtilis, S. epidermidis, L. } \\
\text { monocytogenes, S. choleraesuis, MRSA, Pasteurella } \\
\text { avium, Salmonella enteritidis }\end{array}$ & $(34,36,45,47,59-61,84)$ \\
\hline & Antifungal & $\begin{array}{l}\text { C. albicans, Cryptococcus neoformans, C. tropicalis, } \\
\text { Candida krusei }\end{array}$ & $(34,36)$ \\
\hline PMAP-37 & Antibacterial & $\begin{array}{l}\text { E. coli, S. typhimurium, P. aeruginosa, B. megaterium, } \\
\text { S. aureus, B. subtilis, S. epidermidis, L. } \\
\text { monocytogenes, S. choleraesuis, P. avium }\end{array}$ & $(38,48-50)$ \\
\hline
\end{tabular}

tissues, and difficulty producing drug resistance $(7,73)$. PMAPs have high antimicrobial activity against bacteria and fungi (35, $36,38,71,84)$, and they have certain inhibitory effects on tumors and parasites (94-96). At present, scholars worldwide have designed several new modified peptides through the modification of primary structure and secondary structure. Compared with the original peptides, the modified peptides have higher antimicrobial activity, better therapeutic effect, and lower toxicity. PAMPs with high efficacy and broad antimicrobial spectrum are unlikely to develop drug resistance in the future, thereby presenting new options in terms of developing alternatives to antibiotics and clinical treatment. In addition, PMAP genes may be introduced into animals to confer specific disease resistance by genetic engineering techniques. PMAPs can be applied in animal husbandry as feed additives, resulting in less reliance on traditional antibiotics. However, the antibacterial activity of natural PMAPs is not ideal as PMAPs are easily degraded in vivo. Importantly, the mechanism of how PMAPs bind to intracellular targets and inhibit cell wall, DNA, RNA, and protein synthesis is not well-understood. Further studies on the relationship between structure and activity as well as the mechanism of action are needed to provide an adequate theoretical basis for the design of safe, stable, and efficient AMPs. Many drugs in clinical trials have some type of chemical modification to improve their druggability to improve the possibility of clinical application (7).

Among the structural optimization strategies for PMAPs, amino acid modification and fatty acid modification have been the most effective ways to improve their biological activity. In addition, the application of nanotechnology to modify
AMPs or the use of hydrogel-encapsulated AMPs to make hydrogel preparations and the production of controlled drug release systems are also effective modification strategies $(97,98)$. Currently, studies of PMAPs using these two techniques have not been reported. Therefore, nanomodification and hydrogel preparation may be a new research direction for PMAPs in the future. In summary, continuous research on structural modifications of PMAPs and their mechanisms of action will help to limit the impact of antimicrobial resistance and reduce concerns about the public health hazards caused by antimicrobial resistance.

\section{AUTHOR CONTRIBUTIONS}

SS and TS conducted the literature review and wrote the manuscript. YL and LC revised the manuscript. CW and CL revised the manuscript and administrated the project. All authors read and approved the final manuscript.

\section{FUNDING}

This work was supported by the National Natural Science Foundation of China (U2004151 and 31802159) and the $\mathrm{PhD}$ Start-up Fund of Henan University of Science and Technology (13480071).

\section{ACKNOWLEDGMENTS}

We thank American Journal Experts (AJE) for editing the manuscript. 


\section{REFERENCES}

1. Abushaheen MA, Muzaheed, Fatani AJ, Alosaimi M, Mansy W, George M, et al. Antimicrobial resistance, mechanisms and its clinical significance. Dis Mon. (2020) 66:100971. doi: 10.1016/j.disamonth.2020.100971

2. Konreddy AK, Rani GU, Lee K, Choi Y. Recent drug-repurposing-driven advances in the discovery of novel antibiotics. Curr Med Chem. (2018) 26:5363-88. doi: 10.2174/0929867325666180706101404

3. Aslam B, Wang W, Arshad MI, Khurshid M, Muzammil S, Rasool MH, et al. Antibiotic resistance: a rundown of a global crisis. Infect Drug Resist. (2018) 11:1645-58. doi: 10.2147/IDR.S173867

4. Ferri M, Ranucci E, Romagnoli P, Giaccone V. Antimicrobial resistance: a global emerging threat to public health systems. Crit Rev Food Sci Nutr. (2017) 57:2857-76. doi: 10.1080/10408398.2015.1077192

5. Mobarki N, Almerabi B, Hattan A. Antibiotic resistance crisis. Int J Med Dev Ctries. (2019) 3:561-4. doi: 10.24911/IJMDC.51-1549060699

6. Ahmad M, Khan AU. Global economic impact of antibiotic resistance: a review. J Glob Antimicrob Resist. (2019) 19:3136. doi: 10.1016/j.jgar.2019.05.024

7. Browne K, Chakraborty S, Chen R, Willcox MD, Black DS, Walsh WR, et al. A new era of antibiotics: the clinical potential of antimicrobial peptides. Int $J$ Mol Sci. (2020) 21:7047. doi: 10.3390/ijms21197047

8. Wang $\mathrm{CH}$, Hsieh YH, Powers ZM, Kao CY. Defeating antibiotic-resistant bacteria: exploring alternative therapies for a post-antibiotic era. Int J Mol Sci. (2020) 21:1-8. doi: 10.3390/ijms21031061

9. Machowska A, Stålsby Lundborg C. Drivers of irrational use of antibiotics in Europe. Int J Environ Res Public Health. (2018) 16:27. doi: 10.3390/ijerph16010027

10. Fry DE. Antimicrobial peptides. Surg Infect. (2018) 19:80411. doi: 10.1089/sur.2018.194

11. Mwangi J, Hao X, Lai R, Zhang Z-Y. Antimicrobial peptides: new hope in the war against multidrug resistance. Zool Res. (2019) 40:488505. doi: 10.24272/j.issn.2095-8137.2019.062

12. Hultmark D, Steiner H, Rasmuson T, Boman HG. Insect immunity. Purification and properties of three inducible bactericidal proteins from hemolymph of immunized pupae of hyalophora cecropia. Eur J Biochem. (1980) 106:7-16.

13. Raheem N, Straus SK. Mechanisms of action for antimicrobial peptides with antibacterial and antibiofilm functions. Front Microbiol. (2019) 10:2866. doi: 10.3389/fmicb.2019.02866

14. Boparai JK, Sharma PK. Mini review on antimicrobial peptides, sources, mechanism and recent applications. Protein Pept Lett. (2020) 27:416. doi: 10.2174/0929866526666190822165812

15. Wang $\mathrm{G}$. The antimicrobial peptide database provides a platform for decoding the design principles of naturally occurring antimicrobial peptides. Protein Sci. (2020) 29:8-18. doi: 10.1002/pro.3702

16. Wang Z, Wang G. APD: the antimicrobial peptide database. Nucleic Acids Res. (2004) 32:D590-2. doi: 10.1093/nar/gkh025

17. Wang G. Improved methods for classification, prediction, and design of antimicrobial peptides. Methods Mol Biol. (2015) 1268:43-66. doi: 10.1007/978-1-4939-2285-7_3

18. Wódz K, Brzezińiska-Błaszczyk E. Cathelicidins - endogenous antimicrobial peptides. Postepy Biochem. (2015) 61:93-101.

19. Bals R, Wilson JM. Cathelicidins-a family of multifunctional antimicrobial peptides. Cell Mol Life Sci. (2003) 60:711-20. doi: 10.1007/s00018-003-2186-9

20. Guang H-J, Li Z, Wang Y-P, Lai R, Yu H-N. Progress in cathelicidins antimicrobial peptides research. Zool Res. (2012) 33:523-6. doi: 10.3724/SP.J.1141.2012.05523

21. Wang Y, Wang M, Shan A, Feng X. Avian host defense cathelicidins: structure, expression, biological functions, and potential therapeutic applications. Poult Sci. (2020) 99:6434-45. doi: 10.1016/j.psj.2020.09.030

22. Sang Y, Blecha F. Porcine host defense peptides: expanding repertoire and functions. Dev Comp Immunol. (2009) 33:33443. doi: 10.1016/j.dci.2008.05.006

23. Azari M, Asad S, Mehrnia MR. Heterologous production of porcine derived antimicrobial peptide PR-39 in Escherichia coli using SUMO and intein fusion systems. Protein Expr Purif. (2020) 169:105568. doi: $10.1016 /$ j.pep. 2020.105568
24. Veldhuizen EJA, Schneider VAF, Agustiandari $H$, van Dijk A, Tjeerdsma-van Bokhoven JLM, Bikker FJ, et al. Antimicrobial and immunomodulatory activities of PR-39 derived peptides. PLOS ONE. (2014) 9:e95939. doi: 10.1371/journal.pone.0095939

25. Zhang L, Guo D, Liu Y, Shao Y, Wang Y, Xu Y, et al. Probiotic Lactobacillus casei expressing porcine antimicrobial peptide PR39 elevates antibacterial activity in the gastrointestinal tract. Can J Microbiol. (2016) 62:9619. doi: 10.1139/cjm-2016-0130

26. Choi M-K, Le MT, Cho H, Soundrarajan N, Jeon H, Park CK, et al. Defining the genetic relationship of protegrin-related sequences and the in vivo expression of protegrins. FEBS J. (2014) 281:5420-31. doi: 10.1111/febs.13072

27. Lehrer RI, Ganz T. Endogenous vertebrate antibiotics. Defensins, protegrins, and other cysteine-rich antimicrobial peptides. Ann N Y Acad Sci. (1996) 797:228-39. doi: 10.1111/j.1749-6632.1996.tb52963.x

28. Wessely-Szponder J, Majer-Dziedzic B, Smolira A. Analysis of antimicrobial peptides from porcine neutrophils. J Microbiol Methods. (2010) 83:812. doi: 10.1016/j.mimet.2010.07.010

29. Wang Y, Johansson J, Griffiths WJ. Characterisation of variant forms of prophenin: mechanistic aspects of the fragmentation of proline-rich peptides. Rapid Commun Mass Spectrom. (2000) 14:2182202. doi: 10.1002/1097-0231(20001215)14:23<2182::AID-RCM151>3.0.CO;2-7

30. Huynh E, Akhtar N, Li J. Efficient production of recombinant protegrin-1 from pichia pastoris, and its antimicrobial and in vitro cell migration activity. Front Microbiol. (2018) 9:2300. doi: 10.3389/fmicb.2018.02300

31. Baumann A, Démoulins T, Python S, Summerfield A. Porcine cathelicidins efficiently complex and deliver nucleic acids to plasmacytoid dendritic cells and can thereby mediate bacteria-induced IFN- $\alpha$ responses. J Immunol. (2014) 193:364-71. doi: 10.4049/jimmunol.1303219

32. Boman HG. Peptide antibiotics and their role in innate immunity. Annu Rev Immunol. (1995) 13:61-92. doi: 10.1146/annurev.iy.13.040195.000425

33. Veldhuizen EJA, Scheenstra MR, Tjeerdsma-van Bokhoven JLM, Coorens M, Schneider VAF, Bikker FJ, et al. Antimicrobial and immunomodulatory activity of PMAP-23 derived peptides. Protein Pept Lett. (2017) 24:60916. doi: 10.2174/0929866524666170428150925

34. Scocchi M, Zelezetsky I, Benincasa M, Gennaro R, Mazzoli A, Tossi A. Structural aspects and biological properties of the cathelicidin PMAP-36. FEBS J. (2005) 272:4398-406. doi: 10.1111/j.1742-4658.2005.04852.x

35. Kim S, Lee DG. Role of calcium in reactive oxygen speciesinduced apoptosis in Candida albicans: an antifungal mechanism of antimicrobial peptide, PMAP-23. Free Radic Res. (2019) 53:8-17. doi: 10.1080/10715762.2018.1511052

36. Lyu Y, Yang Y, Lyu X, Dong N, Shan A. Antimicrobial activity, improved cell selectivity and mode of action of short PMAP-36-derived peptides against bacteria and Candida. Sci Rep. (2016) 6:27258. doi: 10.1038/srep27258

37. Bussalleu E, Sancho S, Briz MD, Yeste M, Bonet S. Do antimicrobial peptides PR-39, PMAP-36 and PMAP-37 have any effect on bacterial growth and quality of liquid-stored boar semen? Theriogenology. (2017) 89:23543. doi: 10.1016/j.theriogenology.2016.11.017

38. Tossi A, Scocchi M, Zanetti M, Storici P, Gennaro R PMAP-37, a novel antibacterial peptide from pig myeloid cells. cDNA cloning, chemical synthesis and activity. Eur J Biochem. (1995) 228:941-6. doi: 10.1111/j.1432-1033.1995.tb2 0344.x

39. Travis SM, Anderson NN, Forsyth WR, Espiritu C, Conway BD, Greenberg EP, et al. Bactericidal activity of mammalian cathelicidin-derived peptides. Infect Immun. (2000) 68:2748-55. doi: 10.1128/IAI.68.5.2748-2755.2000

40. Linde CM, Hoffner SE, Refai E, Andersson M. In vitro activity of PR39, a proline-arginine-rich peptide, against susceptible and multi-drugresistant Mycobacterium tuberculosis. J Antimicrob Chemother. (2001) 47:57580. doi: 10.1093/jac/47.5.575

41. Agerberth B, Lee JY, Bergman T, Carlquist M, Boman HG, Mutt V, et al. Amino acid sequence of PR-39. Isolation from pig intestine of a new member of the family of proline-arginine-rich antibacterial peptides. Eur J Biochem. (1991) 202:849-54. doi: 10.1111/j.1432-1033.1991.tb16442.x

42. Shai Y. Mechanism of the binding, insertion and destabilization of phospholipid bilayer membranes by alpha-helical antimicrobial and cell nonselective membrane-lytic peptides. Biochim Biophys Acta. (1999) 1462:5570. doi: 10.1016/S0005-2736(99)00200-X 
43. Liu Y, Shen T, Chen L, Zhou J, Wang C. Analogs of the cathelicidinderived antimicrobial peptide PMAP-23 exhibit improved stability and antibacterial activity. Probiotics Antimicrob Proteins. (2020) 13:273-86. doi: 10.1007/s12602-020-09686-Z

44. Shen T, Chen L, Liu Y, Shi S, Liu Z, Cai K, et al. Decanoic acid modification enhances the antibacterial activity of PMAP-23RI-Dec. Eur J Pharm Sci. (2020) 157:105609. doi: 10.1016/j.ejps.2020.105609

45. Zhou J, Liu Y, Shen T, Chen L, Zhang C, Cai K, et al. Antimicrobial activity of the antibacterial peptide PMAP-36 and its analogues. Microb Pathog. (2019) 136:103712. doi: 10.1016/j.micpath.2019.103712

46. Liu Y, Yan Z, Chai J, Zhou J, Wang C. Antimicrobial activity of the antibacterial peptide PMAP-GI24 and its analogs. Int J Pept Res Ther. (2020) 26:231731. doi: 10.1007/s10989-020-10026-6

47. Liu Y, Li S, Shen T, Chen L, Zhou J, Shi S, et al. N-terminal myristoylation enhanced the antimicrobial activity of antimicrobial peptide PMAP-36PW. Front Cell Infect Microbiol. (2020) 10:450. doi: 10.3389/fcimb.2020.00450

48. Zhou J, Liu Y, Shen T, Chen L, Zhang C, Cai K, et al. Enhancing the antibacterial activity of PMAP-37 by increasing its hydrophobicity. Chem Biol Drug Des. (2019) 94:1986-99. doi: 10.1111/cbdd.13601

49. Zhou J, Chen L, Liu Y, Shen T, Zhang C, Liu Z, et al. Antimicrobial peptide PMAP-37 analogs: increasing the positive charge to enhance the antibacterial activity of PMAP-37. J Pept Sci. (2019) 25:e3220. doi: 10.1002/psc.3220

50. Chen L, Shen T, Liu Y, Zhou J, Shi S, Wang Y, et al. Enhancing the antibacterial activity of antimicrobial peptide PMAP-37(F34-R) by cholesterol modification. BMC Vet Res. (2020) 16:419. doi: 10.1186/s12917-020-02630-x

51. Huan Y, Kong Q, Mou H, Yi H. Antimicrobial peptides: classification, design, application and research progress in multiple fields. Front Microbiol. (2020) 11:582779. doi: 10.3389/fmicb.2020.582779

52. Haney EF, Mansour SC, Hancock REW. Antimicrobial peptides: an introduction. Methods Mol Biol. (2017) 1548:322. doi: 10.1007/978-1-4939-6737-7_1

53. Lee T-H, Hall KN, Aguilar M-I. Antimicrobial peptide structure and mechanism of action: a focus on the role of membrane structure. Curr Top Med Chem. (2016) 16:25-39. doi: 10.2174/15680266156661507031 21700

54. Guerreiro CIPD, Fontes CMGA, Gama M, Domingues L. Escherichia coli expression and purification of four antimicrobial peptides fused to a family 3 carbohydrate-binding module (CBM) from Clostridium thermocellum. Protein Expr Purif. (2008) 59:161-8. doi: 10.1016/j.pep.2008. 01.018

55. Park K, Oh D, Kim Y, Yub Shin S, Hahm KS. Structural studies of porcine myeloid antibacterial peptide PMAP-23 and its analogues in DPC micelles by NMR spectroscopy. Biochem Biophys Res Commun. (2002) 290:20412. doi: 10.1006/bbrc.2001.6173

56. Kang JH, Shin SY, Jang SY, Kim KL, Hahm KS. Effects of tryptophan residues of porcine myeloid antibacterial peptide PMAP23 on antibiotic activity. Biochem Biophys Res Commun. (1999) 264:281-6. doi: 10.1006/bbrc.1999.1510

57. Yang S-T, Jeon J-H, Kim Y, Shin SY, Hahm K-S, Kim J Il. Possible role of a PXXP central hinge in the antibacterial activity and membrane interaction of PMAP-23, a member of cathelicidin family. Biochemistry. (2006) 45:177584. doi: 10.1021/bi051524k

58. Bocchinfuso G, Palleschi A, Orioni B, Grande G, Formaggio F, Toniolo C, et al. Different mechanisms of action of antimicrobial peptides: insights from fluorescence spectroscopy experiments and molecular dynamics simulations. J Pept Sci. (2009) 15:550-8. doi: 10.1002/psc.1144

59. Wang Z, Zhang L, Wang J, Wei D, Shi B, Shan A. Synergistic interaction of PMAP-36 and PRW4 with aminoglycoside antibiotics and their antibacterial mechanism. World J Microbiol Biotechnol. (2014) 30:31218. doi: 10.1007/s11274-014-1739-4

60. Scheenstra MR, van den Belt M, Tjeerdsma-van Bokhoven JLM, Schneider VAF, Ordonez SR, van Dijk A, et al. Cathelicidins PMAP-36, LL-37 and CATH-2 are similar peptides with different modes of action. Sci Rep. (2019) 9:4780. doi: 10.1038/s41598-019-41246-6

61. Lv Y, Wang J, Gao H, Wang Z, Dong N, Ma Q, et al. Antimicrobial properties and membrane-active mechanism of a potential $\alpha$-helical antimicrobial derived from cathelicidin PMAP-36. PLoS ONE. (2014) 9:e86364. doi: 10.1371/journal.pone.0086364
62. Wang L, Zhang H, Jia Z, Ma Q, Dong N, Shan A. In vitro and in vivo activity of the dimer of PMAP-36 expressed in Pichia pastoris. J Mol Microbiol Biotechnol. (2014) 24:234-40. doi: 10.1159/000365572

63. Ma QQ, Jiao WJ, Wang ZY, Wu CX, Shan AS, Wang YB, et al. Tissue specificity and species superiority of cathelicidin gene expression in Chinese indigenous Min pigs. Livest Sci. (2014) 161:36-40. doi: 10.1016/j.livsci.2014.01.004

64. Guilhelmelli F, Vilela N, Albuquerque P, Derengowski LdS, Silva-Pereira I, Kyaw CM. Antibiotic development challenges: the various mechanisms of action of antimicrobial peptides and of bacterial resistance. Front Microbiol. (2013) 4:353. doi: 10.3389/fmicb.2013.00353

65. Wang S, Zeng X, Yang Q, Qiao S. Antimicrobial peptides as potential alternatives to antibiotics in food animal industry. Int J Mol Sci. (2016) 17:603. doi: 10.3390/ijms17050603

66. Oren Z, Shai Y. Mode of action of linear amphipathic alphahelical antimicrobial peptides. Biopolymers. (1998) 47:451-63. doi: 10.1002/(SICI)1097-0282<451::AID-BIP4>3.0.CO;2-F (1998) 47:6

67. Matsuzaki K. Membrane permeabilization mechanisms. Adv Exp Med Biol. (2019) 1117:9-16. doi: 10.1007/978-981-13-3588-4_2

68. Shai Y, Oren Z. From "carpet" mechanism to de-novo designed diastereomeric cell-selective antimicrobial peptides. Peptides. (2001) 22:1629-41. doi: 10.1016/S0196-9781(01)00498-3

69. Wu Q, Patočka J, Kuča K. Insect antimicrobial peptides, a mini review. Toxins. (2018) 10:461. doi: 10.3390/toxins10110461

70. Kim S, Lee DG. PMAP-23 triggers cell death by nitric oxide-induced redox imbalance in Escherichia coli. Biochim Biophys acta Gen Subj. (2019) 1863:1187-95. doi: 10.1016/j.bbagen.2019.04.014

71. Zanetti M, Storici P, Tossi A, Scocchi M, Gennaro R. Molecular cloning and chemical synthesis of a novel antibacterial peptide derived from pig myeloid cells. J Biol Chem. (1994) 269:7855-8. doi: 10.1016/S0021-9258(17)37128-4

72. Kim J-Y, Park S-C, Yoon M-Y, Hahm K-S, Park Y. C-terminal amidation of PMAP-23: translocation to the inner membrane of gram-negative bacteria. Amino Acids. (2011) 40:183-95. doi: 10.1007/s00726-010-0632-1

73. Liu Y, Shi J, Tong Z, Jia Y, Yang B, Wang Z. The revitalization of antimicrobial peptides in the resistance era. Pharmacol Res. (2020) 163:105276. doi: 10.1016/j.phrs.2020.105276

74. Fillion M, Valois-Paillard G, Lorin A, Noël M, Voyer N, Auger M. Membrane interactions of synthetic peptides with antimicrobial potential: effect of electrostatic interactions and amphiphilicity. Probiotics Antimicrob Proteins. (2015) 7:66-74. doi: 10.1007/s12602-014-9177-z

75. Park IY, Cho JH, Kim KS, Kim Y-B, Kim MS, Kim SC. Helix stability confers salt resistance upon helical antimicrobial peptides. J Biol Chem. (2004) 279:13896-901. doi: 10.1074/jbc.M311418200

76. Ahmed TAE, Hammami R. Recent insights into structure-function relationships of antimicrobial peptides. J Food Biochem. (2019) 43:e12546. doi: 10.1111/jfbc. 12546

77. Tajbakhsh M, Karimi A, Tohidpour A, Abbasi N, Fallah F, Akhavan MM. The antimicrobial potential of a new derivative of cathelicidin from Bungarus fasciatus against methicillin-resistant Staphylococcus aureus. J Microbiol. (2018) 56:128-37. doi: 10.1007/s12275-018-7444-5

78. Shin SY, Kang SW, Lee DG, Eom SH, Song WK, Kim JI. CRAMP analogues having potent antibiotic activity against bacterial, fungal, and tumor cells without hemolytic activity. Biochem Biophys Res Commun. (2000) 275:9049. doi: 10.1006/bbrc.2000.3269

79. Carvalho A de O, Gomes VM. Plant defensins-prospects for the biological functions and biotechnological properties. Peptides. (2009) 30:1007-20. doi: 10.1016/j.peptides.2009.01.018

80. Asthana N, Yadav SP, Ghosh JK. Dissection of antibacterial and toxic activity of melittin: a leucine zipper motif plays a crucial role in determining its hemolytic activity but not antibacterial activity. J Biol Chem. (2004) 279:55042-50. doi: 10.1074/jbc.M408881200

81. Wiradharma N, Sng MYS, Khan M, Ong Z-Y, Yang Y-Y. Rationally designed $\alpha$-helical broad-spectrum antimicrobial peptides with idealized facial amphiphilicity. Macromol Rapid Commun. (2013) 34:74-80. doi: 10.1002/marc.201200534

82. Nagaoka I, Kuwahara-Arai K, Tamura H, Hiramatsu K, Hirata M. Augmentation of the bactericidal activities of human cathelicidin CAP18/LL37-derived antimicrobial peptides by amino acid substitutions. Inflamm Res Off. (2005) 54:66-73. doi: 10.1007/s00011-004-1323-8 
83. Koh JJ, Lin S, Beuerman RW, Liu S. Recent advances in synthetic lipopeptides as anti-microbial agents: designs and synthetic approaches. Amino Acids. (2017) 49:1653-77. doi: 10.1007/s00726-017-2476-4

84. Storici P, Scocchi M, Tossi A, Gennaro R, Zanetti M. Chemical synthesis and biological activity of a novel antibacterial peptide deduced from a pig myeloid cDNA. FEBS Lett. (1994) 337:303-7. doi: 10.1016/0014-5793(94)80214-9

85. Arias M, Piga KB, Hyndman ME, Vogel HJ. Improving the activity of trp-rich antimicrobial peptides by Arg/Lys substitutions and changing the length of cationic residues. Biomolecules. (2018) 8:19. doi: 10.3390/biom8020019

86. Wu Y, Huang R, Jin J-M, Zhang L-J, Zhang H, Chen H-Z, et al. Advances in the study of structural modification and biological activities of anoplin. Front Chem. (2020) 8:519. doi: 10.3389/fchem.2020.00519

87. Kamysz E, Sikorska E, Jaśkiewicz M, Bauer M, Neubauer D, Bartoszewska S, et al. Lipidated analogs of the LL-37-derived peptide fragment KR12structural analysis, surface-active properties and antimicrobial activity. Int $J$ Mol Sci. (2020) 21:887. doi: 10.3390/ijms21030887

88. Li C, Zhu C, Ren B, Yin X, Shim SH, Gao Y, et al. Two optimized antimicrobial peptides with therapeutic potential for clinical antibiotic-resistant Staphylococcus aureus. Eur J Med Chem. (2019) 183:111686. doi: 10.1016/j.ejmech.2019.111686

89. Ramezanzadeh M, Saeedi N, Mesbahfar E, Farrokh P, Salimi F, Rezaei A. Design and characterization of new antimicrobial peptides derived from aurein 1.2 with enhanced antibacterial activity. Biochimie. (2020) 181:4251. doi: 10.1016/j.biochi.2020.11.020

90. Ciociola T, Giovati L, Conti S, Magliani W, Santinoli C, Polonelli L. Natural and synthetic peptides with antifungal activity. Future Med Chem. (2016) 8:1413-33. doi: 10.4155/fmc-2016-0035

91. Delattin N, De Brucker K, De Cremer K, Cammue BPA, Thevissen K. Antimicrobial peptides as a strategy to combat fungal biofilms. Curr Top Med Chem. (2017) 17:604-12. doi: 10.2174/1568026616666160713142228

92. Lee DG, Kim DH, Park Y, Kim HK, Kim HN, Shin YK, et al. Fungicidal effect of antimicrobial peptide, PMAP-23, isolated from porcine myeloid against Candida albicans. Biochem Biophys Res Commun. (2001) 282:5704. doi: 10.1006/bbrc.2001.4602
93. Lee DG, Kim P Il, Park Y, Woo E-R, Choi JS, Choi C-H, et al. Design of novel peptide analogs with potent fungicidal activity, based on PMAP-23 antimicrobial peptide isolated from porcine myeloid. Biochem Biophys Res Commun. (2002) 293:231-8. doi: 10.1016/S0006-291X(02)00 222-X

94. Park Y, Jang S-H, Lee DG, Hahm K-S. Antinematodal effect of antimicrobial peptide, PMAP-23, isolated from porcine myeloid against Caenorhabditis elegans. J Pept Sci. (2004) 10:304-11. doi: 10.1002/psc.518

95. Wharton DA, Jenkins T. Structure and chemistry of the eggshell of a nematode (Trichuris suis). Tissue Cell. (1978) 10:42740. doi: 10.1016/S0040-8166(16)30338-X

96. Shin SY, Kang JH, Jang SY, Kim KL, Hahm KS. Structure and antibiotic activity of a porcine myeloid antibacterial peptide, PMAP-23 and its analogues. J Biochem Mol Biol. (2000) 33:49-53. Available online at: http://www. koreascience.kr/article/JAKO200034755186443

97. Carratalá JV, Serna N, Villaverde A, Vázquez E, Ferrer-Miralles N. Nanostructured antimicrobial peptides: the last push towards clinics. Biotechnol Adv. (2020) 44:107603. doi: 10.1016/j.biotechadv.2020.107603

98. Yang G, Huang T, Wang Y, Wang H, Li Y, Yu K, et al. Sustained release of antimicrobial peptide from self-assembling hydrogel enhanced osteogenesis. J Biomater Sci Polym Ed. (2018) 29:1812-24. doi: 10.1080/09205063.2018.1504191

Conflict of Interest: The authors declare that the research was conducted in the absence of any commercial or financial relationships that could be construed as a potential conflict of interest.

Copyright (c) 2021 Shi, Shen, Liu, Chen, Wang and Liao. This is an open-access article distributed under the terms of the Creative Commons Attribution License (CC $B Y)$. The use, distribution or reproduction in other forums is permitted, provided the original author(s) and the copyright owner(s) are credited and that the original publication in this journal is cited, in accordance with accepted academic practice. No use, distribution or reproduction is permitted which does not comply with these terms. 